\title{
Cost-effectiveness modeling of abatacept versus other biologic agents in DMARDS and anti-TNF inadequate responders for the management of moderate to severe rheumatoid arthritis
}

\author{
Anthony Russell • Ariel Beresniak • Louis Bessette • \\ Boulos Haraoui • Proton Rahman • Carter Thorne • \\ Ross Maclean • Danielle Dupont
}

Received: 5 May 2008 /Revised: 2 October 2008 / Accepted: 18 November 2008 /Published online: 17 December 2008

(C) Clinical Rheumatology 2008

\begin{abstract}
To assess the cost-effectiveness of abatacept compared to different biologic treatment strategies for moderate to severe rheumatoid arthritis based on current medical practices in Canada. A model was constructed to assess the cost-effectiveness of various biologic treatments over a 2-year time horizon, using two effectiveness endpoints: "low disease activity state" (LDAS) and "remission". Abatacept, as first biologic agent after an inadequate response to DMARDs, provides greater treatment success rate for achieving LDAS (29.4\% versus $15.6 \%$ ) and remission ( $14.8 \%$ versus $5.2 \%$ ), and appears significantly more cost-effective compared to the sequential use of antiTNF agents $(p<0.001)$. Abatacept, as second biologic agent after an inadequate response to one anti-TNF agent, provides greater treatment success rate for achieving LDAS (17.1\% versus $10.2 \%)$ and remission $(7.4 \%$ versus $3.9 \%)$
\end{abstract}

A. Russell

University of Alberta,

Edmonton, Canada

A. Beresniak $(\bowtie)$

LIRAES, Paris-Descartes University,

Paris, France

e-mail: aberesniak@datamining-international.com

A. Beresniak

Data Mining International,

Geneva, Switzerland

L. Bessette

Centre Hospitalier Universitaire de Québec-CHUL,

Québec, Canada

B. Haraoui

University of Montreal,

Montreal, Canada and appears significantly more cost-effective compared to the sequential use of anti-TNF agents $(p<0.001)$. Abatacept is a cost-effective strategy in patients with an inadequate response to DMARDs or to one anti-TNF agent.

Keywords Abatacept $\cdot$ Anti-TNF therapy Costeffectiveness $\cdot$ Modeling $\cdot$ Rheumatoid Arthritis

\section{Introduction}

During the past decade, the therapeutic management of patients with Rheumatoid Arthritis (RA) has undergone significant evolution. There has been a shift in the management of RA towards beginning therapy immediately with diseasemodifying anti-rheumatic drugs (DMARDs) in order to

P. Rahman

St. Clare's Mercy Hospital,

St. John's, Newfoundland, Canada

C. Thorne

Southlake Regional Health Center, University of Toronto, Toronto, Canada

R. Maclean

Outcomes Research, Bristol-Myers Squibb,

Princeton, NJ, USA

D. Dupont

Bristol-Myers Squibb International Corporation,

Braine L'Alleud, Belgium 
control disease activity, to reduce functional impairment and to prevent irreversible changes in cartilage and bone [1]. However, in the long term, patients treated with DMARDs may show deterioration in radiographs and joint function, indicating the need for improved therapies. To address these unmet needs, biologic agents were developed and provide treatment alternatives for patients with an inadequate response to DMARDs. The first biologic agents introduced on the market were tumor necrosis factor antagonists (antiTNFs) and include etanercept, infliximab, and adalimumab.

Although anti-TNF therapies combined with methotrexate (MTX) have shown improved efficacy in clinical trials compared to DMARDs alone, a significant proportion of patients are not effectively treated with these biologic therapies, either due to lack or loss of efficacy and/or to adverse events. In controlled clinical trials, $25 \%$ to $50 \%$ of patients fail to achieve the minimum $20 \%$ improvement in ACR criteria following anti-TNF treatment, and only a small proportion of patients achieves a 50 or $70 \%$ improvement [2-7]. In addition, some studies have shown that treatment discontinuation rates with anti-TNF treatment approaches $30 \%$ at 1 year, due to lack or loss of efficacy and/or to adverse events [8,9]. There is also emerging evidence of a risk of decreased efficacy with the sequential use of anti-TNF therapies [8]. For these patients, this represents an unmet medical need for which new therapeutic options are required. Furthermore, direct and indirect medical costs associated with RA are significant [10]. Thus, alternative treatment strategies have been developed to address the above unmet needs [11, 12].

To assist decision-making, the introduction of a new biologic option, such as abatacept, requires an assessment of its value in the context of existing therapies. The value of a new therapeutic option is defined by its clinical benefits and by conducting cost-effectiveness analyses which assess and compare the effectiveness of different technologies in relation with their corresponding overall treatment costs.

The arrival of abatacept, the first of a new class of biologic agents for rheumatoid arthritis known as selective co-stimulation modulators, offers a new therapeutic alternative for patients with moderate to severe active RA and an inadequate response to one or more DMARDs and/or to TNF antagonists $[11,12]$.

\section{Materials and methods}

\section{Model framework}

In the absence of well-designed head-to-head clinical trials assessing and comparing the effectiveness of various biologic agents, economic evaluations of health care technologies use models to synthesize evidence from multiple sources in order to estimate short, mid-, or long-term costs relative to the outcomes of various therapeutic strategies. These models allow the representation of current treatment patterns, therapeutic strategies, and treatment switches, and take into account the overall impact of both treatment success and failure relative to each technology being assessed [13].

The objective of this simulation model was to assess the cost-effectiveness of abatacept vs. anti-TNF therapies in patients with moderate to severe RA and with an inadequate response to one or more DMARDs and/or anti-TNF agents. The analysis includes direct costs and was thus conducted using the perspective of the public payer.

The defined population entering this model is composed of patients with moderate to severe RA with an inadequate response to DMARDs, eligible for biologic therapy. In this model, patients achieving treatment success (defined as either achieving a low disease-activity state (LDAS) or remission) are maintained on existing therapy for up to 2 years. Only those with an inadequate response to a biologic therapy are switched to a subsequent biologic agent, with decision to switch made at 6 months intervals in case of an inadequate response. To assess the cost-effectiveness of abatacept used as first biologic therapy in patients with an inadequate response to DMARDs and as second biologic therapy in patients with an inadequate response to a first anti-TNF agent, the comparator was defined as a successive trial of anti-TNF therapies based on the most established treatment pattern in Canada at time of model development. The selection of treatment options was based on local medical practices and the most robust drug prescription surveys available. As rituximab was not reimbursed for rheumatoid arthritis in Canada at time of model development, it was not considered a valid comparator for the purpose of this analysis.

In this model, each strategy being assessed and compared is composed of different biologic agents used successively following an inadequate response to the previous treatment. The model also includes two clinically relevant effectiveness endpoints (either achieving LDAS or remission), which are defined as the most desirable and clinically relevant outcomes in RA [14-16]. To take into account all potential therapeutic scenarios and clinical outcomes, 14 decision trees similar to the one presented in Fig. 1 were developed.

The decision tree illustrated in Fig. 1 is composed of 45 health states (branches) and 30 transition probabilities. Each decision tree uses the following set of parameters: Success rate (LDAS or remission rate) over 2 years and confidence interval (CI), Corresponding medical costs over 2 years and CI, Mean cost-effectiveness ratios (mean costs divided by mean effectiveness) and CI, and Incremental cost-effectiveness ratios (ICER: costs difference divided by effectiveness difference) of one strategy versus an alternative strategy.

These decision trees were designed as "simulation models" and programmed to take into account the entire 


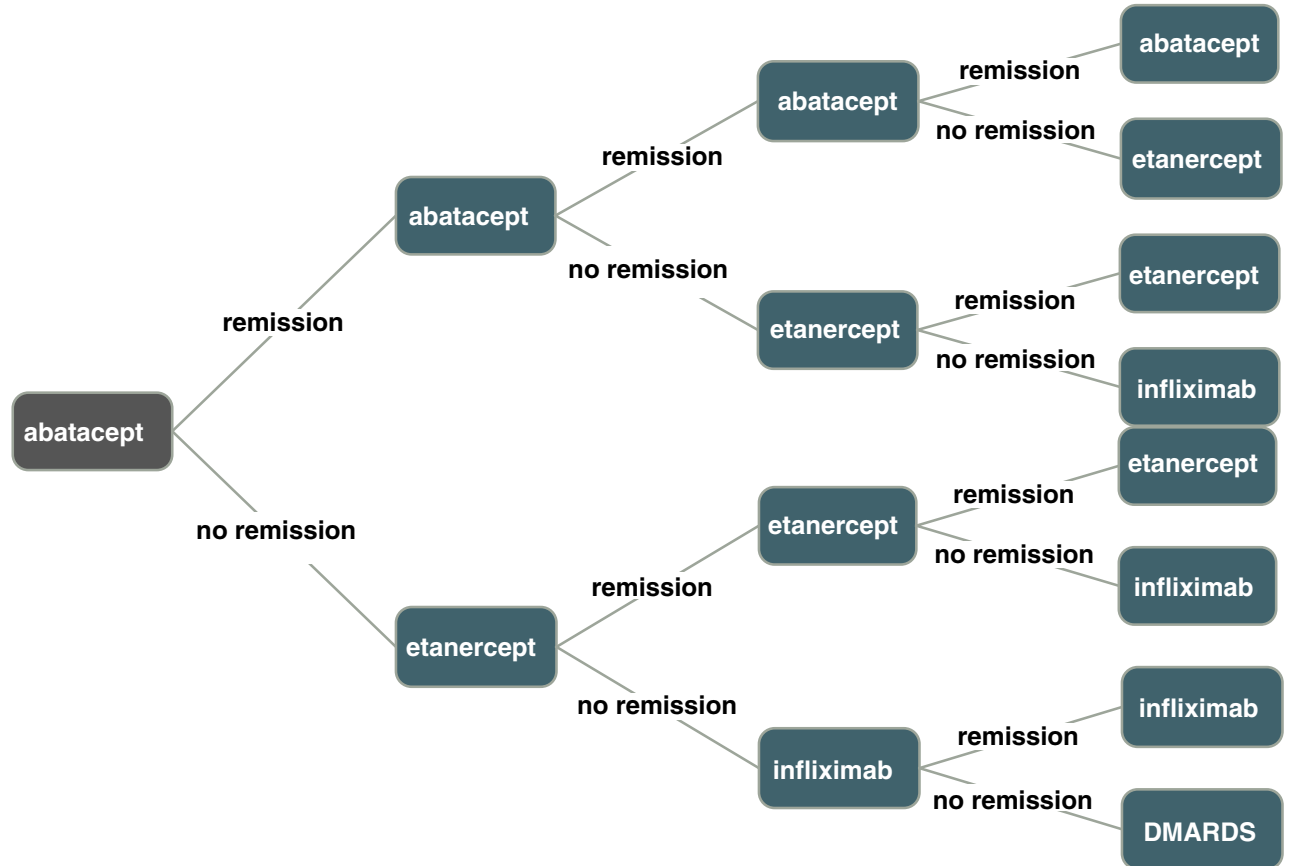

Fig. 1 Decision tree framework - abatacept used as first biologic option over 2 years (success endpoint: remission)

distribution costs and distribution effectiveness for each pre-defined parameter according to specific distribution laws. For simulation models consuming large amounts of computer processing time, powerful workstations with parallel-processors and adapted programming languages (Dscript language-DecisionPro ${ }^{\circledR}$ software) were used.

Other model assumptions were considered consistent with clinical practices: the same treatment continues as long as it is efficacious; decision to switch treatment may occur only in case of lack of success to treatment for all causes (e.g., lack or loss of efficacy, adverse events, intolerance, etc); the model allows switches to occur every 6 months.

Regarding costing aspects, this cost-effectiveness analysis uses the perspective of the public payer and considers direct medical costs. However, given the significant indirect costs associated with RA, the cost-effectiveness results can be considered conservative from a societal perspective.

\section{Effectiveness endpoints}

The primary objective when treating RA is to control inflammatory activity of the disease, and if possible, to achieve remission [14-16]. Thus, clinical remission is considered as the most desirable clinical outcome [14]. Control of disease activity in RA is therefore an important treatment goal for preventing the progression of joint damage and functional disability, and their clinical and economic consequences [17, 18]. Remission has been most often defined using the Disease-Activity Score (DAS) which is a composite index that includes variables such as the number of tender and swollen joints, erythrocyte sedimentation rate (ESR), and the patient's assessment of disease activity. This index facilitates the quantification of disease activity and provides a more reliable overall estimate than would each individual measurement [16]. While the original DAS44 score is based on the assessment of 44 joints, a DAS index using a low number of joint counts (DAS28) was developed and validated and the response criteria appear to be as valid as the ones with larger number of joint counts. Hence for simplicity, the reduced number of joint counts (DAS28) is now preferred in standard clinical practice [16]. Newer and simpler indices are also gaining in acceptance such as the Simple Disease-Activity Index (SDAI) and the Clinical Disease-Activity Index (CDAI).

Given that remission and LDAS represent the primary treatment objectives in RA, for the purpose of this costeffectiveness analysis, treatment effectiveness was defined as either achieving disease remission (DAS28<2.6) or LDAS (DAS28 $\leq 3.2$ ).

The DAS induction data used to populate the model are from published pivotal trials and the maintenance data (up to 2 years) are from either published trials or clinical trial analyses [11, 12, 19, 20]. For abatacept, clinical trial evidence included all clinical evidence available and published at time of model development. This included 12 months data in patients with an inadequate response to DMARDs (Phase II and Phase III AIM trial) $[12,20]$ and 6 months data in patients with an inadequate response to anti-TNF therapies (ATTAIN trial) [11]. Two-year extension maintenance effectiveness data from both pivotal trials was 
Table 1 Induction success rates $(\%$ patients achieving low disease-activity state and remission)

\begin{tabular}{lccc}
\hline Biologic therapy & Low disease-activity state & Remission & Source \\
\hline $\begin{array}{l}\text { Abatacept (DMARD inadequate } \\
\text { responders) }\end{array}$ & 0.40 & 0.261 & Kremer 2005 \\
$\begin{array}{l}\text { Abacept (anti-TNF inadequate } \\
\text { responders) }\end{array}$ & 0.183 & 0.111 & ATTAIN Study \\
$\begin{array}{l}\text { Etanercept (DMARD inadequate } \\
\text { responders) }\end{array}$ & 0.45 & 0.303 & $\begin{array}{c}\text { TEMPO Study } \\
\text { (van der Heijde 2006) }\end{array}$ \\
\hline
\end{tabular}

used confirming long-term and sustained clinical benefits in both patient populations responding adequately [21, 22].

The safety of abatacept in patients with RA was evaluated in a large, randomized, placebo-controlled trial [23] and in the ATTAIN and AIM trials and extension studies [21, 22].

As indicated in Table 1, the induction effectiveness data (effectiveness of one specific biologic class appearing in the sequence for the first time) is from the etanercept [19] and from the abatacept clinical trials available at time of model development [11, 12, 20].

The maintenance effectiveness data beyond the first 6month cycle (i.e., effectiveness of one specific biologic class maintained after the first cycle and for one or more subsequent 6 months cycles) in DMARDS inadequate responders is again from the TEMPO trial for etanercept and from the AIM trial for abatacept. Considering the TEMPO trial [19], effectiveness data comes from the third arm of the trial combining etanercept and methotrexate. This is consistent with the effectiveness data extracted from the abatacept trials (AIM and ATTAIN) where abatacept was used in combination with DMARDs.

The maintenance effectiveness data in anti-TNF inadequate responders is from the ATTAIN trial for abatacept [11]. In the absence of well-designed controlled clinical trials assessing the effectiveness of anti-TNFs in anti-TNF inadequate responders, the ATTAIN study results were objectively applied to each anti-TNF switch, using the assumption of a $10 \%$ reduction in effectiveness after each switch. This assumption reflects the unanimous opinion of the clinical experts as well as recent clinical evidence reporting that switching anti-TNF agents is often associated with a lower efficacy and/or increased rate of treatment discontinuation [8, 24-27].

As economic modeling considers active treatment arms, placebo adjustment was not performed. This is because the objective of most cost-effectiveness models is to consider "effectiveness" data (total observed effectiveness including placebo effect, concomitant drugs effects such as MTX, etc.), and not only "efficacy" data (effect of one active agent considered in isolation) in order to reflect real-life settings. However, the proposed methodology does take into account study population variability (differences in inclusion and exclusion criteria, protocols, etc.) in simulating all parameters distributions (extensive probabilistic sensitivity analysis using Monte Carlo simulation techniques).

The model calculates as an effectiveness outcome the overall effectiveness of each entire sequence of biologic strategies. The final outcome of the model is a computation and comparison of different sequences of biologic strategies to reflect current medical practice, which consists of switching biologic therapies in case of an inadequate response to the previous one.

\section{Cost analysis}

Drug costs are expressed in Canadian dollars and were calculated based on approved product monographs in Canada and 2006 list prices (Table 2). Where relevant, the number of units required per year was calculated based on the average body weight in Canada estimated at $75 \mathrm{~kg}$ for the targeted age group of 45-64 years [28].

As per approved label, abatacept is administered over a 30-min i.v. infusion (no pre-medication required) at 2 and 4 weeks after the first infusion, and every 4 weeks thereafter. Assuming a $75 \mathrm{~kg}$ average body weight in Canada and based on abatacept fixed dose regimen per body weight range $(<60 \mathrm{~kg}=500 \mathrm{mg}, \geq 60$ and $\leq 100 \mathrm{~kg}=$ $750 \mathrm{mg}$, and $>100 \mathrm{~kg}=1,000 \mathrm{mg}$ ) [Health Canada Product Monograph 2006], the analysis considers an average dose

Table 2 Estimated annual costs of therapy

\begin{tabular}{lcc}
\hline $\begin{array}{l}\text { Estimated annual cost of } \\
\text { biological therapies }\end{array}$ & Year 1 (CAN \$) & Year 2 (CAN \$) \\
\hline $\begin{array}{l}\text { Abatacept (250 mg vial) } \\
\text { Adalimumab (40 mg pre-filled }\end{array}$ & $\$ 18,480$ & $\$ 17,160$ \\
$\quad$ syringe) & $\$ 17,680$ & $\$ 17,680$ \\
Etanercept (25 mg vial) & $\$ 20,445$ & $\$ 18,200$ \\
Infliximab (100 mg vial) & $\$ 18,330$ \\
\hline
\end{tabular}

Based on Health Canada product monographs and published literature

${ }^{\text {a }}$ Reported on a 6-month basis in the cost-effectiveness model

${ }^{\mathrm{b}}$ Assuming an average body weight of $75 \mathrm{~kg}$

${ }^{\mathrm{c}}$ Includes loading dose Year 1

${ }^{\mathrm{d}}$ Assuming $3 \mathrm{mg} / \mathrm{kg}$ for the first three doses (at week 0, 2, and 6) then an average of $4 \mathrm{mg} / \mathrm{kg}$ every 8 weeks thereafter (i.e., $50 \%$ of patients requiring $3 \mathrm{mg} / \mathrm{kg}$ and $50 \%$ requiring $5 \mathrm{mg} / \mathrm{kg}$ ) 
of $750 \mathrm{mg}(3 \times 250 \mathrm{mg}$ vials $)$ per infusion. Infusion costs were not included because in Canada, infliximab and abatacept were administered in participating rheumatology and infusion clinics (and even at home for abatacept) and hence, were not incurred by the public payer.

Direct medical costs per DAS score categories were assessed based on a Canadian cost survey $[29,30]$. In this study, data were collected from 253 adult patients throughout the province of Ontario. Detailed resource utilization items of the following cost categories were collected: visits to health professionals [family physician, specialist (non-surgical reported separately from surgical visits), allied health, dentist], laboratory tests or investigation (X-ray, CT, MRI, ultrasound, ECG, other laboratory, bone density), hospitalizations, prescribed drugs (arthritis [not including anti-TNF or co-stimulation modulator], anti-hypertensive, gastro-protective, other), home care, transportation services. adaptive aids/other devices.

Direct medical cost data were analyzed for 138 patients for whom four DAS components were complete. Data for these RA patients were tabulated and categorized within the DAS framework by threshold scores (Table 3) [29].

\section{Uncertainty management}

For each uncertain variable (one that has a range of possible values), probability distribution and confidence intervals were selected by the authors. A lognormal distribution shape was selected to program costing variability. The main advantage of lognormal distribution is the zero origin, which allows the simulation of costing data.

A beta distribution shape was selected to program transition probabilities variability. Beta distribution is a continuous probability distribution with the function defined on the interval $[0,1]$, which allows to simulate probability data (always between 0 and $1=100 \%$ ). For example, as different studies were used to provide the efficacy data in the model, one could argue that patient populations would not be similar and consequently,

Table 3 Total 6-month direct costs per DAS categories

\begin{tabular}{lrrr}
\hline DAS category & Number of patients & \multicolumn{2}{l}{$\begin{array}{l}\text { Direct 6 months costs } \\
\text { (CAN\$) }\end{array}$} \\
\cline { 3 - 4 } & & Mean & \multicolumn{1}{c}{ s.d. } \\
\hline $\mathrm{DAS} \leq 2.6$ & 4 & 1,052 & 235 \\
$\mathrm{DAS}>2.6$ & 134 & 1,994 & 1,352 \\
$\mathrm{DAS} \leq 3.2$ & 11 & 1,764 & 2,069 \\
$\mathrm{DAS}>3.2$ & 127 & 1,984 & 1,271 \\
\hline
\end{tabular}

W Katchamart, X Li, C Bombardier, Direct and Indirect Costs of Rheumatoid Arthritis are Strongly Correlated with Disease Activity and Functional Status, 2006 ACR/ARHP Poster Session B treatment responses would not be directly comparable. Taking into account the entire distribution of effectiveness parameters, this potential variability was addressed and managed by the model.

In addition, as recommended in the 2006 Canadian Agency for Drugs and Technologies in Health (CADTH) guidelines for economic evaluation of health technologies (www.cadth.ca), an extensive probabilistic sensitivity analysis using Monte Carlo simulations was used to manage the uncertainty of the model. Monte Carlo simulations randomly select a value from the defined possibilities (range and shape of the distribution) of each parameter and then recalculates the outcomes [31]. Confidence intervals of outcomes have been calculated using 5,000 Monte Carlo simulations. By screening all uncertain parameter values to construct outcome confidence intervals, this approach is considered a robust sensitivity analysis ("probabilistic sensitivity analysis") [31-34].

\section{Results}

Reference case: 2-year treatment with up to three successive biologic agents (in case of an inadequate response to the previous biologic agent)

\section{Achieving LDAS after an inadequate response to DMARDs}

Considering the current anti-TNF sequential strategy of reference:

- Etanercept $\rightarrow$ Infliximab $\rightarrow$ Adalimumab $\rightarrow$ DMARDs

the following therapeutic strategies including abatacept were simulated:

- Abatacept $\rightarrow$ Etanercept $\rightarrow$ Infliximab $\rightarrow$ DMARDs

- Etanercept $\rightarrow$ Abatacept $\rightarrow$ Infliximab $\rightarrow$ DMARDs.

Considering patients with an inadequate response to DMARDs entering the model, compared to anti-TNF therapies, the lowest cost biologic strategy was abatacept used as the first biologic agent. This strategy was dominant (i.e., both more effective and less costly), providing 13.8\% greater probability of achieving LDAS than sequential antiTNF therapy (29.4\% vs. $15.6 \%)$ with an overall RA-related cost-saving of $\$ 730$ (\$39,759 vs. $\$ 40,489)$ over 2 years (Table 4).

Using the same patient population at entry, abatacept used as second biologic after an inadequate response to one anti-TNF agent (etanercept) was compared to sequential anti-TNF therapies. Abatacept was cost-effective, providing $3.7 \%$ greater probability of achieving LDAS $(19.3 \%$ vs. $15.6 \%)$ at an additional cost of $\$ 463(\$ 40,952$ vs. $\$ 40,489)$ over the 2 -year period, representing an incre- 
Table 4 Cost-effectiveness of abatacept vs. anti-TNF strategies for achieving low diseaseactivity state and remission in patients with an inadequate response to DMARDs

\begin{tabular}{lccccc}
\hline Sequential biologic strategies & $\begin{array}{c}\text { Treatment } \\
\text { Cost }(2 \text { years })\end{array}$ & $\begin{array}{l}\text { Treatment } \\
\text { success }\end{array}$ & $\begin{array}{l}\text { Mean cost- } \\
\text { effectiveness }\end{array}$ & $p$ value & ICER \\
\hline $\begin{array}{c}\text { Low disease-activity state } \\
\text { Abatacept-etanercept- } \\
\text { infliximab-DMARDs }\end{array}$ & $\$ 39,759$ & 0.294 & $\$ 136,730$ & $<0.0001$ & Dominant \\
$\begin{array}{c}\text { Etanercept-abatacept- } \\
\text { infliximab-DMARDs }\end{array}$ & $\$ 40,952$ & 0.193 & $\$ 213,872$ & $<0.0001$ & $\$ 12,514$ \\
$\begin{array}{c}\text { Etanercept-infliximab- } \\
\text { adalimumab-DMARDs } \\
\begin{array}{c}\text { Remission } \\
\text { Abatacept-etanercept- } \\
\text { infliximab-DMARDs }\end{array}\end{array}$ & $\$ 40,489$ & 0.156 & $\$ 261,943$ & - & - \\
$\begin{array}{c}\text { Etanercept-abatacept- } \\
\text { infliximab-DMARDs }\end{array}$ & $\$ 38,061$ & 0.148 & $\$ 263,240$ & $<0.0001$ & Dominant \\
$\begin{array}{c}\text { Etanercept-infliximab- } \\
\text { adalimumab-DMARDs }\end{array}$ & $\$ 39,154$ & 0.087 & $\$ 463,689$ & $<0.0001$ & $\$ 16,829$ \\
\hline
\end{tabular}

mental cost-effectiveness ratio (ICER) of $\$ 12,514$ per additional case of LDAS gained (Table 4). Thus, abatacept used as first biologic appears to be less costly and to provide greater probability of achieving LDAS than using abatacept as second biologic agent. Mean cost-effectiveness ratio also showed statistically significant lower cost for achieving LDAS with abatacept as first biologic agent $(p<0.0001)$

\section{Achieving remission after an inadequate response to DMARDs}

Using the sequential biologic strategies cited above, the same simulations were conducted with disease remission as the effectiveness endpoint. Again, the lowest cost strategy was abatacept used as the first biologic agent (Table 4). This strategy was dominant, providing 9.6\% greater probability of remission compared to sequential anti-TNF therapy (14.8\% vs. 5.2\%) with an overall RA-related costsaving of $\$ 504(\$ 38,061$ vs. $\$ 38,565)$. Using the same population of patients with an inadequate response to DMARDs at entry, abatacept used as second biologic after inadequate response to one anti-TNF agent (etanercept) was compared to sequential anti-TNF therapies. Abatacept was cost-effective, providing $3.5 \%$ greater probability of remission $(8.7 \%$ vs. $5.2 \%)$ at an additional cost of $\$ 589(\$ 39,154$ vs. \$38,565) over the 2-year period, representing an incremental cost-effectiveness ratio (ICER) of $\$ 16,829$ per additional remission gained. Again, abatacept used as first biologic was found to be less costly overall and to provide greater probability of remission than abatacept used as second biologic option (Table 4).

Mean cost-effectiveness ratio also showed statistically significant lower cost for achieving remission with abatacept used as first biologic agent $(p<0.0001)$.
Achieving LDAS or remission after an inadequate response to one anti-TNF agent

To assess the cost-effectiveness of abatacept in patients with an inadequate response to one anti-TNF agent, alternate modeling was performed where this time the model only enrolled patients with an inadequate response to a first anti-TNF agent (etanercept). This alternate model was conducted to allow a more direct comparison of abatacept as second biologic agent versus sequential antiTNF therapies in this patient population. The model was programmed setting a failure to an initial 6-month treatment with etanercept so that all patients were switched to either abatacept or infliximab as the second biologic option, followed by infliximab and adalimumab, respectively.

Under this alternate modeling approach, compared to cycled anti-TNF therapies, abatacept used as second biologic agent after an inadequate response to one antiTNF agent was cost-effective, providing 6.9\% additional treatment success rate for achieving LDAS (17.1\% vs. $10.2 \%$-Table 5) and 3.5\% additional treatment success rates for achieving remission (7.4\% vs. 3.9\% - Table 5), at an incremental cost-effectiveness ratio (ICER) of $\$ 20,377$ per additional case of LDAS and $\$ 26,400$ per additional remission, respectively.

\section{Discussion}

When conducting economic evaluations, it is recommended to use data analogous to real-life benefits and a clinical pathway consistent with the practice of the country in which the analysis is conducted [33]. Economic evaluations should also reflect clinical practice and ideally, should include disease-specific clinical outcomes [34]. 
Table 5 Cost-effectiveness of abatacept vs. anti-TNF strategies for achieving low diseaseactivity state and remission in patients with an inadequate response to one anti-TNF

\begin{tabular}{lccccc}
\hline Sequential biologic strategies & $\begin{array}{l}\text { Treatment cost } \\
(2 \text { years })\end{array}$ & $\begin{array}{l}\text { Treatment } \\
\text { success }\end{array}$ & $\begin{array}{l}\text { Mean cost- } \\
\text { effectiveness }\end{array}$ & $p$ value & ICER \\
\hline $\begin{array}{c}\text { Low disease-activity state } \\
\text { Etanercept-abatacept- } \\
\text { infliximab-DMARDs }\end{array}$ & $\$ 38,596$ & 0.171 & $\$ 231,654$ & $<0.0001$ & $\$ 20,377$ \\
$\begin{array}{c}\text { Etanercept-infliximab- } \\
\text { adalimumab-DMARDs }\end{array}$ & $\$ 37,190$ & 0.102 & $\$ 389,999$ & - & \\
$\begin{array}{c}\text { Remission } \\
\text { Etanercept-abatacept- } \\
\text { infliximab-DMARDs }\end{array}$ & $\$ 37,281$ & 0.074 & $\$ 541,925$ & $<0.0001$ & $\$ 26,400$ \\
$\begin{array}{c}\text { Etanercept-infliximab- } \\
\text { adalimumab-DMARDs }\end{array}$ & $\$ 36,357$ & 0.039 & $\$ 1,131,085$ & - & - \\
\hline
\end{tabular}

As achieving LDAS or remission represent primary therapeutic objectives and most desirable clinical outcomes in RA [14-16], achieving these treatment outcomes is becoming an important measure by which to compare different treatment strategies [35].

Since response relative to baseline may have less significance as duration of a particular therapy increases, describing the disease status in absolute terms may be more relevant in this circumstance. As suggested by the Outcome Measures in Rheumatology Clinical Trials (OMERACT) group, this can be done by a continuous absolute measure such as the DAS and by counting the proportion of patients classified as being in remission or in a low disease-activity state [34]. Thus, achieving LDAS or remission was used as the most clinically relevant effectiveness endpoints in this analysis. The OMERACT group also suggests that comparative studies in RA consider not one therapeutic agent but rather a sequence of therapies over the long term, i.e., a therapeutic sequence strategy. Modeling of therapeutic sequences is of particular importance in evaluating the cost-effectiveness of a chronic disease therapy to help identify the most clinically relevant population for a new drug, and where it may be best positioned within an established therapeutic sequence [34]. While realistic sequences should be modeled, depending on the clinical setting, research documenting the type of sequences used by rheumatologists is also lacking.

Comparing across clinical trials is always a difficult task as populations and methodologies are not necessarily similar. This is why such approaches must be undertaken with extreme caution. However, robust meta-analyses are nowadays considered as part of evidence-based medicine and simulation models provide interesting additional techniques to combine and compare data from different sources (clinical trials, literature, reports, observational data, etc.). In the present model, data variability was managed using probabilistic sensitivity analyses and validated assumptions to integrate data from heterogeneous sources. Hence, modeling approaches provide methodolog- ical frameworks for conclusions to be interpreted in the context of their specific underlying assumptions.

For the purpose of this cost-effectiveness analysis (CEA), in the absence of comparative trials, the most established anti-TNF treatment pattern in Canada at time of model development was used as comparator, based on local medical practice and market research data, and validated by an expert panel six expert rheumatologists.

Clinical trials duration represents another challenge when assessing long-term impact. Most experts agree that models should include at least one-year time horizon as modeling clinical benefit beyond trial duration is a contentious element that often requires relative outcomes measures which may impede the comparability of economic evaluations $[33,34]$. To best reflect clinical practice in this model, in accordance with clinical experts, the time horizon was set at 2 years with the possibility to switch treatment at 6-month intervals in case of inadequate response.

Another type of economic evaluation to consider could have been to conduct a cost-utility analysis (CUA) which requires the use of "utility" scores (preference assessment) to generate Quality Adjusted Life Years (QALY) as a potential composite assessment indicator. These type of economic evaluations are regularly published in the area of rheumatology [36-38] but are often inadequately presented as "cost-effectiveness" studies. This may add to the confusion regarding two methods that are neither equivalent nor interchangeable. While real cost-effectiveness analyses compare costs with an effectiveness criteria expressed in natural unit of clinical outcome (such as "disease remission" as a valid clinical success criteria), cost-utility analyses compare costs with a QALY indicator derived from patient preferences to construct a "utility" score. However, there is increasing evidence that the QALY indicator used in RA may lead to divergent or inconsistent results depending on the utility assessment method used [33, 39-44]. As reported in the CADTH economic guidelines, CUAs and related preferences assessment instruments often produce different scores for the same health state. 
Another reported concern is that the QALY is not a clinically meaningful indicator and may not discriminate between conditions with different severity. Furthermore, most cost-utility models expressed in cost/QALY use a long-term time horizon [such as lifetime in the Brennan model [38] and very fragile assumptions regarding longterm efficacy. Given these limitations, a CEA was preferred for the purpose of this analysis where results are presented in terms of costs per unit of clinical effectiveness expressed in natural outcome units over a 2-year time horizon, consistent with clinical trials evidence, and without having to speculate nor to project non-evidence-based long-term efficacy over lifetime.

However, improvement in quality of life is of paramount importance in the management of RA and using a real costeffectiveness demonstration (expressed in cost per clinical outcome) should not reduce the significance of the improvement in quality of life as an important and relevant additional outcome. When compared to traditional DMARDs, abatacept was shown in the AIM and ATTAIN trials to statistically significantly improve all quality of life domains in patients with an inadequate response to DMARDs and/or to anti-TNF therapy $[11,12]$.

Clinical guidelines currently recommend the use of biologic agents after an inadequate response to traditional DMARDs. Abatacept introduces a new class of biologic agents with a distinct mechanism of action than anti-TNF agents. Incremental clinical and economic value is therefore expected given the risk of reduced efficacy and high discontinuation rates reported with the sequential use of anti-TNF therapies [8, 24-27], and the well-reported issue of dose escalation with infliximab in more than $50 \%$ of patients [45-51].

The uniqueness and strength of this cost-effectiveness analysis is that it uses robust and clinically relevant outcomes as effectiveness endpoints (i.e., remission or LDAS as RA treatment goals) to compare therapeutic strategies which represent current medical practice for the management of RA, including biologic treatment switches.

\section{Conclusion}

This advanced cost-effectiveness modeling is the first assessing various biologic strategies based on current medical practice for the management of RA in Canada. The results show that when used as first biologic agent in patients with moderate to severe RA and with an inadequate response to DMARDs, abatacept appears to be dominant (i.e., overall less costly and more effective) compared to anti-TNF therapies, consistently showing significantly lower treatment cost and better probability of achieving treatment success, defined as either LDAS or remission. The results also show that when used as second biologic agent in patients with an inadequate response to a first anti-TNF agent, abatacept is cost-effective compared to anti-TNF therapies. This CEA being based on direct costs, the cost-effectiveness results are likely understated from a societal perspective. Once comparative long-term clinical evidence becomes available, similar modeling approaches will be useful to simulate long-term costs, effectiveness, and cost-effectiveness.

Acknowledgment We are grateful to Dr Claire Bombardier, MD, FRCPC, Mount Sinai Hospital, University of Toronto (Canada) for providing the data on the Ontario cohort for the calculation of the cost per DAS categories.

Disclosures Anthony Russell, Ariel Beresniak, Louis Bessette, Boulos Haraoui, Proton Rahman and Carter Thorne have received fees and honorarium to attend Steering Committee meetings and to defray travel expenses reimbursement from Bristol-Myers Squibb.

Ross Maclean and Danielle Dupont are employees of Bristol-Myers Squibb.

Source of funding The work has been sponsored by Bristol-Myers Squibb International.

\section{References}

1. Pisetsky D, St Clair E (2001) Progress in the treatment of rheumatoid arthritis. JAMA 286:2787-2790

2. Weinblatt M, Kremer J, Bankhurst A, Bulpitt K, Fleischmann R, Fox R, Jackson C, Lange M, Burge D (1999) A trial of etanercept, a recombinant tumor necrosis factor receptor: $\mathrm{Fc}$ fusion protein, in patients with rheumatoid arthritis receiving methotrexate. N Engl J Med 340:253-259

3. Weinblatt M, Keystone E, Furst D, Moreland L, Weisman M, Birbara C, Teoh L, Fischkoff S, Chartash E (2003) Adalimumab, a fully human anti-tumor necrosis factor alpha monoclonal antibody, for the treatment of rheumatoid arthritis in patients taking concomitant methotrexate: the ARMADA trial. Arthritis Rheum 48:35-45

4. Lipsky P, van der Heijde D, St Clair E, Furst D, Breedveld F, Kalden J, Smolen J, Weisman M, Emery P, Feldmann M, Harriman G, Maini R, Group A-TNFTiRAwCTS (2000) Infliximab and methotrexate in the treatment of rheumatoid arthritis. Anti-Tumor Necrosis Factor Trial in Rheumatoid Arthritis with Concomitant Therapy Study Group. N Engl J Med 343:1594-1602

5. Redlich K, Schett G, Steiner G, Hayer S, Wagner EJSS (2003) Rheumatoid arthritis therapy after tumor necrosis factor and interleukin-1 blockade. Arthritis Rheum 48:3308-3319

6. Klinkhoff A (2004) Biological agents for rheumatoid arthritis: targeting both physical function and structural damage. Drugs 64:1267-1283

7. Smolen J, Steiner G (2003) Therapeutic strategies for rheumatoid arthritis. Nature Reviews 2

8. Hyrich K, Lunt M, Watson K, Symmons D, Silman A (2007) Outcomes after switching from one anti-tumor necrosis factor agent to a second anti-tumor necrosis factor agent in patients with rheumatoid arthritis. Arthritis Rheum 56:13-20

9. Flendrie M, Creemers M, Welsing PMJ, den Broeder AA, van Riel PLCM (2003) Survival during treatment with tumour 
necrosis factor blocking agents in rheumatoid arthritis. Ann Rheum Dis 62:30-33

10. Pugner KM, Scott DI, Holmes JW, Hieke K (2000) The costs of rheumatoid arthritis: an international long-term view. Semin Arthritis Rheum 29:305-320

11. Genovese M, Becker J, Schiff M, Luggen M, Sherrer Y, Kremer J, Birbara C, Box J, Natarajan K, Nuamah I, Li T, Aranda R, Hagerty D, Dougados M (2005) Abatacept for rheumatoid arthritis refractory to tumor necrosis factor alpha inhibition. N Engl J Med 353:1114-1123

12. Kremer J, Genant H, Moreland L, Russel A et al (2006) Effects of abatacept in patients with methotrexate-resistant active rheumatoid arthritis: a randomized trial. Ann Intern Med 144:865-876

13. Stahl J (2008) Modelling methods for pharmacoeconomics and health technology assessment, an overview and guide. Pharmacoeconomics 26:131-148

14. Makinen H, Kautiainen H, Hannonen P, Tuulikki S (2005) Frequency of remissions in early rheumatoid arthritis defined by 3 sets of criteria. A 5-year followup study. J Rheumatol 32:796800

15. American College of Rheumatology (2002) Guidelines for the management of rheumatoid arthritis. Arthritis Rheum 46:328346

16. Fransen J, Van Riel PLCM (2005) The disease activity score and the EULAR response criteria. Clin Exp Rheumatol 23:S93-S99

17. Welsing PMJ, van Gestel AM, Swinkels HL, Keimeney LALM, Van Riel PLCM (2001) The relationship between disease activity, joint destruction and functional capacity over the course of rheumatoid arthritis. Arthritis Rheum 44:2009-2017

18. Welsing PMJ, Severens JL, Hartman M, van Gestel AM, van Riel PLCM, Laan RFJM (2006) The initial validation of a Markov model for the economic evaluation of (new) treatments for rheumatoid arthritis. Pharmacoeconomics 24:1011-1020

19. Van der Heijde D, Klareskog L, Rodriguez-Valverde V, Codreanu C, Bolosiu H, Melo-Gomes J, Tornero-Molina J, Wajdula J, Pedersen R, Fatenejad S, Investigators ftTS (2006) Comparison of etanercept and methotrexate, alone and combined, in the treatment of rheumatoid arthritis two-year clinical and radiographic results from the TEMPO study, a double-blind, randomized trial. Arthritis Rheum 54:1063-1074

20. Kremer J, Dougados M, Emery P, Durez P, Sibilia J, Shergy W, Steinfeld S, Tindall E, Becker J, Li T, Nuamah I, Aranda R, Moreland L (2005) Treatment of rheumatoid arthritis with the selective costimulation modulator abatacept -12 month results of a phase II double-blind, randomized, placebo-controlled trial. Arthritis Rheum 52:2263-2271 Aug

21. Genovese M, Schiff M, Luggen M, Becker J, Aranda R, Teng J, Li T, Schmidely N, Le Bars M, Dougados M (2007) Efficacy and safety of the selective co-stimulation modulator abatacept following 2 years of treatment in patients with rheumatoid arthritis and an inadequate response to anti-TNF therapy. Ann Rheum Dis (online) Oct 5:doi:10.1136/ard.2007.074773

22. Hervey P, Keam S (2006) Abatacept. Biodrugs 20:53-61

23. Weinblatt M, Combe B, Covucci A, Aranda R, Becker J, Keystone E (2006) Safety of the selective costimulation modulator abatacept in rheumatoid arthritis patients receiving background biologic and nonbiologic disease-modifying antirheumatic drugs. A one year randomized placebo-controlled study. Arthritis Rheum 54:2807-2816

24. Haraoui B, Cameron L, L'Archevêque J, Ouellet M, Choquette D, Raynauld J (2007) Clinical response to a second or third anti-TNF agent after discontinuation of the first. Implications for therapeutic decision-making. 4th Canadian Association for Population Therapeutics (CAPT) Congress Halifax

25. Navaro F, Marsal S, Gomez-Reino J, Perez-Pampon E (2006) EULAR response and drug survival in clinical practice in RA patients treated with anti-TNF therapy: results of an observational, prospective, cohort study in Spain. Ann Rheum Dis 65:333

26. Van Vollenhoven R, Cullinane Carli C, Bratt J, Klareskog L (2006) Six-year report of the STURE registry for biologicals in rheumatology: satisfactory overall results, but plenty of room for improvement. Ann Rheum Dis 65:511

27. Gomez-Reino J, Carmona L, Group tB (2006) Switching TNF antagonists in patients with chronic arthritis: an observational study of 488 patients over a four-year period. Arthritis Research \& Therapy 8:(doi:10.1186/ar1881); http://arthritis-research.com/con tent/1188/1181/R1129

28. Canadian Fitness and Lifestyle Research Institute (1995) Physical Activity Monitor. http://wwwdflrica/cflri/pa/surveys/95survey/ 95surveyhtml

29. Katchamart W, Li X, Bombardier C (2006) Direct and indirect costs of rheumatoid arthritis are strongly correlated with disease activity and functional status. ACR/ARHP 625

30. Maetzel A, Li LC, Pencharz J, Tomlinson G, Bombardier C (2004) The economic burden associated with osteoarthritis, rheumatoid arthritis and hypertension: a comparative study. Ann Rheum Dis 63:395-401

31. Welsing PMJ, Severens JL, Harman M, van Riel PLCM, Laan RFJM (2004) Modeling the 5-year cost-effectiveness of treatment strategies including tumor necrosis factor-blocking agents and leflunomide for treating rheumatoid arthritis in the Netherlands. Arthritis Rheum (Athritis Care \& Research) 51:964-973

32. Emery P (2004) Review of health economics modelling in rheumatoid arthritis. Pharmacoeconomics 22:55-69

33. Bansback N, Regier D, Ara R, Brennan A, Shojania K, Esdaile J, Anis Aslam H, Marra Carlo A (2005) An overview of economic evaluations for drugs used in rheumatoid arthritis: focus on tumour necrosis factor-alpha antagonists. Drugs 65:473-496

34. Maetzel A, Tugwell P, Boers M, Guillemin F, Coyle D, Drummond M, Wong J, Gabriel S, Group obotOER (2003) Economic evaluation of programs or interventions in the management of rheumatoid arthritis: defining a reference case. J Rheumatol 30:891-896

35. Wells G, Boers M, Shea B, Brooks P, Simon L, Strand C, Aletaha D, Anderson J, Bombardier C, Dougados M, Emery P, Felson D, Fransen J, Furst D, Hazes J, Johnson K, Kirwan J, Landewé R, Lassere M, Michaud K, Suarez-Almazor M, Silman A, Smolen J, Van der Heijde D, van Riel P, Wolfe F, Tugwell P (2005) Minimal disease activity for rheumatoid arthritis: a preliminary definition. J Rheumatol 32:2016-2024

36. Lyseng-Williamson KA, Plosker GL (2004) Etanercept: a pharmacoeconmic review of its use in rheumatoid arthritis. Pharmacoeconomics 22:1071-1095

37. Doan QV, Chiou CG, Dubois RW (2006) Review of eight pharmacoeconomic studies of the value of biologic DMARDS (adalimumab, etanercept and infliximab) in the management of rheumatoid arthritis. J Manag Care Pharm 12:555-569

38. Brennan A, Bansback N, Nixon R, Madan J, Harrison M, Watson K, Symmons D (2007) Modelling the cost-effectiveness of TNFalpha antagonists in the management of rheumatoid arthritis: results from the British Society for Rheumatology Biologics Registry. Rheumatology 46:1345-1354

39. Suarez-Almazor M, Conner-Spady B (2001) Rating of arthritis health states by patients, physicians, and the general public. Implications for cost-utility analyses. J Rheumatol 28:648-656

40. Duru G, Auray JP, Beresniak A, Lamure M, Paine A, Nicoloyannis N (2002) Limitations of the methods used for calculating quality-adjusted life-year values. Pharmacoeconomics 20:463-473

41. Mc Gregor M, Caro J (2006) QALYs: Are they helpful to decision makers? Pharmacoeconomics 24:947-952

42. Jorstad I, Kirstiansen I, Uhlig T et al (2005) Performance of four utility measures in 1041 patients with rheumatoid arthritis (RA): 
well correlated but differing widely in valuing health states. Arthritis Rheum 52:S660

43. Ariza-Ariza R, Hernández-Cruz B, Carmona L, Ruiz-Montecinos MD, Ballina J, Navarro-Sarabia F, Group CaQoLiRAS (2006) Assessing utility values in rheumatoid arthritis: a comparison between time trade-off and the EuroQol. Arthritis Rheum (Arthritis Care Res) 55:751-756

44. Beresniak A, Russell A, Haraoui B, Bessette L, Bombardier C, Duru G (2007) Advantages and limitations of utility assessment methods in rheumatoid arthritis. J Rheumatol 34:2193-2200

45. Ariza-Ariza R, Navarro-Sarabia F, Hernández-Cruz B, RodríguezArboleya L, Navarro-Compán V, Toyos J (2007) Dose escalation of the anti-TNF- $\alpha$ agents in patients with rheumatoid arthritis. A systematic review. Rheumatology 46:529-532

46. Haraoui B, Cameron L, Ouellet M, White B (2006) Antiinfliximab antibodies in patients with rheumatoid arthritis who require higher doses of infliximab to achieve or maintain a clinical response. J Rheumatol 33:31-36
47. Wolbink G, Vis M, Lems W, Voskuyl A, de Groot E, Nurmohamed M, Stapel S, Tak P, Aarden L, Dijkmans B (2006) Development of antiinfliximab antibodies and relationship to clinical response in patients with rheumatoid arthritis. Arthritis Rheum 54:711-715

48. Berger A, Edelsberg J, Li T, Maclean J, Oster G (2005) Dose intensification with infliximab in patients with rheumatoid arthritis. Ann Pharmacother 39:2021-2025

49. Stern R, Wolfe F (2004) Infliximab dose and clinical status: results of 2 studies in 1642 patients with rheumatoid arthritis. J Rheumatol 31:1538-1545

50. Fitzcharles M, Clayton D, Menard H (2002) The use of infliximab in academic rheumatology practice: an audit of early clinical experience. J Rheumatol 29:2525-2530

51. Agarwal S, Maier A, Chibnik L, Coblyn J, Fossel A, Lee R, Fanikos J, Fiumara K, Lowry C, Weinblatt M (2005) Pattern of infliximab utilization in rheumatoid arthritis patients at an academic medical center. Arthritis Rheum (Arthritis Care Res) 53:872-878 